\title{
Percepción que tienen las gestantes sobre el cuidado de enfermería en la atención prenatal $^{*}$
}

\author{
Perception of the Pregnant Women about the Nursing Care in the Prenatal Services \\ Percepção das gestantes sobre o cuidado de enfermagem no atendimento pré-natal
}

\author{
Lucy Marcela Vesga Gualdrón ${ }^{\text {a }}$ \\ Universidad Nacional de Colombia, Colombia \\ lmvesgag@unal.edu.co \\ ORCID: http://orcid.org/0000-0003-1575-1782 \\ Carmen Helena Ruiz de Cárdenas \\ Universidad Nacional de Colombia, Colombia \\ ORCID: http://orcid.org/0000-0002-9679-0320
}

DOI: https://doi.org/10.11144/Javeriana.ie21-1.ptgc Redalyc: http://www.redalyc.org/articulo.oa? id $=145257605006$

Fecha de recepción: 30 Octubre 2017 Fecha de aprobación: 18 Julio 2018

\section{Resumen:}

Introducción: Favorecer la adherencia al control prenatal es necesario para mejorar la salud materno-perinatal. El cuidado humano que ofrece enfermería es determinante. Objetivo: Describir la percepción de las mujeres gestantes frente a las competencias técnicas, cognitivas y la capacidad de los profesionales de enfermería de dar cuidado humanizado durante la atención prenatal. Método: Diseño descriptivo de corte trasversal, que empleó una muestra de 150 gestantes, abordadas de manera secuencial, seleccionadas por conveniencia, provenientes de cuatro centros de atención distintos, adscritos a un hospital de Bogotá, Colombia, durante el periodo junio-agosto de 2013. Se empleó la Escala de Cuidado Profesional fundada en la teoría El cuidado para el bienestar del otro, que permite identificar las habilidades técnicas y de cuidado humano. Resultados: El cuidado profesional fue calificado como excelente; sin embargo, existen elementos del cuidado humano que deben reconocerse. La habilidad para permitir la expresión de sentimientos, la escucha y la atención sin reproches o críticas a las condiciones particulares de las mujeres son valoradas de manera positiva y pueden modificar la percepción de la competencia cognitiva del profesional. Conclusiones: Estos atributos del cuidado humano pueden ser determinantes en la adherencia al control prenatal y su importancia debe ser reconocida por los profesionales sanitarios.

Palabras clave: atención prenatal, atención de enfermería, enfermería obstétrica, satisfacción del paciente.

\begin{abstract}
:
Introduction: The human care provided by the nurses is a determining factor in the promotion of the adherence to the prenatal control, which is necessary to improve the maternal-perinatal health. Objective: To describe the perception of pregnant women about the technical and cognitive competences and capability of the nursing professionals to provide a humanized health care during the prenatal services. Method: This work has a cross-sectional descriptive design using a sample of 150 pregnant women. They were recruited sequentially and based on some convenience factors, from four different health care centers belonging to a main hospital in Bogotá, Colombia. They were recruited between June and August 2013. In this process the professional healthcare scale based on the theory Care for the Other's Well-being was used, which allows to identify the technical and human care skills. Results: The professional health care was assessed as excellent. There are however some elements of the human care that should be highlighted: the ability to allow the expression of feelings; the listening and attention without reproaching or criticizing particular conditions in the women. These elements are valued positively and can modify their perception about the professional's cognitive competence. Conclusions: These attributes of the human care can be determining factors for the adherence to the prenatal control; therefore, the public health professional should recognize their importance.
\end{abstract}

Keywords: prenatal health care, nursing care, obstetric nursing, patient's satisfaction.

\footnotetext{
${ }^{\text {a }}$ Autor de correspondencia: lmvesgag@unal.edu.co
} 


\section{Resumo:}

Introdução: Favorecer a adesão ao controlo pré-natal é preciso para melhorar a saúde materno-perinatal. O cuidado humano que oferece a enfermagem é determinante. Objetivo: Descrever a percepção das mulheres gestantes frente às competências técnicas, cognitivas e a capacidade dos profissionais de enfermagem de dar cuidado humanizado durante o atendimento pré-natal. Método: Desenho descritivo de corte transversal que empregou amostra de 150 grávidas, abordadas de maneira sequencial, selecionadas por conveniência, provindo de quatro centros de atendimento diferentes, adscritos a um hospital de Bogotá, Colômbia, durante o período junho-agosto de 2013. A Escala de Cuidado Professional fundada na teoria O cuidado para o bemestar do outrem foi usada permitindo identificar as habilidades técnicas e de cuidado humano. Resultados: O cuidado professional foi qualificado como excelente; no entanto, existem elementos do cuidado humano que devem se reconhecer. A habilidade para permitir a expressão de sentimentos, a escuta e o atendimento sim reproches ou críticas às condições particulares das mulheres são valoradas de maneira positiva e podem modificar a percepção da competência cognitiva do professional. Conclusões: Estes atributos do cuidado humano podem ser determinantes na adesão ao controle pré-natal e sua importância deve ser reconhecida pelos professionais sanitários.

Palavras-chave: atendimento pré-natal, atendimento de enfermagem, enfermagem obstétrica, satisfação do paciente.

\section{Introducción}

El control prenatal es una estrategia mundial que ha mostrado resultados favorables en la disminución de complicaciones maternas y perinatales $(1,2,3,4)$. Su adherencia ha motivado diversos estudios que han identificado, entre otros aspectos, barreras en el acceso a los servicios de atención prenatal. Estos resultados han tenido repercusiones benéficas que trasforman la ejecución de los programas (5,6,7); sin embargo, estudios de tipo cualitativo realizados en distintos países exponen que factores propios del cuidado humano - la percepción materna de sentirse criticada, de ser atendida sin interés en su condición particular o la comunicación inefectiva con los profesionales, por ejemplo- pueden ser motivadores de la inasistencia o el abandono del control prenatal. También mencionan aspectos protectores de la adherencia a la estrategia, como la continuidad del cuidado prenatal, ser atendidas por mujeres y recibir información sobre la gestación, especialmente centrada en los autocuidados maternos y los cuidados con su hijo por nacer $(2,8,9,10)$.

Considerando lo expuesto, se identificó la importancia de realizar una medición objetiva que les permita a las mujeres gestantes evaluar el cuidado profesional que reciben, por supuesto destacando elementos del cuidado humano. En esta investigación se han dejado de lado características de calidad del cuidado obstétrico como la infraestructura, los dispositivos médicos, el acceso a los servicios o el cumplimiento de las guías de manejo y protocolos de atención $(1,11)$. Se dio prioridad, en cambio, al trato sensible y a la posibilidad de establecer una efectiva interrelación con la mujer gestante, que permita una fácil enseñanza y la identificación de las necesidades individuales (12).

De la misma manera, en la percepción de la confianza que puede trasmitir el profesional, de sus habilidades técnicas y conocimientos que dan credibilidad y tranquilidad de la competencia de su desempeño $(3,9,11,12)$. Por lo anterior, el presente estudio tuvo como objetivo describir la percepción de las mujeres gestantes frente al cuidado profesional que ofrece enfermería, centrado en dos aspectos principales: la capacidad de ofrecer cuidado competente, entendido como las habilidades cognitivas, técnicas y procedimentales de las que dispone el profesional en su atención, y el cuidado compasivo que da cuenta de la capacidad del profesional de ofrecer un cuidado sensible, centrado en el reconocimiento de las necesidades individuales. Así mismo, describir la relación que existe entre estos aspectos fundamentales del cuidado profesional. 


\section{Método}

Estudio descriptivo de corte transversal derivado de un estudio metodológico principal; por ello, el tamaño de muestra se calculó en función de la extensión de la escala. Empleó una muestra de 150 mujeres gestantes, diez por cada ítem de la escala (13) abordadas en cuatro unidades primarias de atención (UPA), adscritas al Hospital de Engativá en la ciudad de Bogotá, Colombia, entre junio y agosto de 2013. Las participantes se seleccionaron de manera secuencial. Entre los criterios de inclusión se consideraron: ser mayor de 14 años, en capacidad de asentir su participación en el estudio y asistir al control prenatal por enfermería a una de las cuatro UPA. Como criterio de exclusión estuvo tener discapacidad cognitiva diagnosticada. Esta investigación acogió los principios éticos que establece la Resolución 008430 de 1993 emitida por el Ministerio de Salud. Se obtuvo el consentimiento o asentimiento verbal, respondiendo al principio de autonomía. Del total de las mujeres contactadas, dos se negaron a participar, en cuyo caso se respetó su voluntad. El manejo de datos tuvo en cuenta las disposiciones de la Ley 1581 de 2012, que reglamenta la protección de datos personales.

La escala se aplicó de manera telefónica, cumpliendo con un protocolo de comunicación que garantizó la explicación del objetivo del estudio. Se respetó la confidencialidad de la información recogida, con el uso de códigos numéricos atendiendo al principio de no maleficencia. El promedio de tiempo empleado fue de cuatro minutos y medio por cada participante. Otros aspectos éticos contemplados son el principio de beneficencia, reflejado en la amplia revisión de la literatura que sustenta el planteamiento problemático, pues la importancia del tema es tal que la salud de la mujer gestante y la adherencia a las estrategias que la promueven son asuntos contenidos en los objetivos de desarrollo sostenible. De la misma manera, esta investigación contempla el respeto por las políticas de derechos de autor. El proyecto contó con la aprobación del Comité de Ética de la Facultad de Enfermería de la Universidad Nacional de Colombia y con el aval de la institución de salud.

El instrumento empleado para medir el constructo cuidado profesional fue la Escala de Cuidado Profesional, versión en español de 2013, que se fundamenta en la teoría de mediano rango: el cuidado para el bienestar del otro, de Kristen Swanson. Cuenta con quince ítems de extensión y pruebas métricas de validez facial, de contenido, de constructo y confiabilidad. La consistencia interna que refleja el alfa de Cronbach $(0,907)$ indica una alta cohesión de los ítems de la escala. En su constructo subyacen dos dimensiones: 1) el sanador compasivo, que hace referencia a las habilidades del profesional frente al cuidado integral, sensible y humanizado, y 2) el sanador competente, que evalúa las habilidades técnicas y cognitivas de quien presta el cuidado (1).

No se documentan antecedentes de aplicación telefónica de esta escala en la literatura revisada; sin embargo, existen diversas ventajas con la disposición de estrategias apoyadas en tecnología, entre ellas la disminución de costos y de tiempo en la aplicación; la posibilidad de aclarar a los participantes las dudas de manera inmediata, lo que no se consigue cuando se emplea de forma autoadministrada; así como una mayor tasa de respuesta y diligenciamiento completo de las escalas $(14,15,16)$. Por ello, investigadores que han abordado muestras de mujeres en puerperio han considerado la estrategia telefónica como buena para la salud pública (16).

El análisis estadístico se realizó en el programa SPSS versión 22.0. Las variables ordinales se analizaron con estadísticos como moda, media, máximo, mínimo y distribución de frecuencias; para variables de nivel de intervalo se empleó la desviación estándar. Se comprobó la distribución de los datos mediante la prueba de Kolmogorov-Smirnov y se emplearon pruebas no paramétricas de varianza entre grupos, para observar si existían diferencias entre las variables en cada uno de los centros incluidos en la recolección. Adicionalmente, se empleó la prueba de correlación de Spearman para explorar asociaciones entre las variables (13). Las pruebas fueron realizadas estableciendo un intervalo de confianza (IC) del $95 \%$. 


\section{Resultados}

De manera general, la muestra se caracterizó por una alta frecuencia de gestantes adolescentes, un bajo nivel de escolaridad, ligado a la condición de vulnerabilidad. Las madres participantes de esta investigación tenían, en promedio, 22,4 años; no se incluyeron madres menores de 14 años, por su imposibilidad de asentimiento. Más del 40\% fueron gestantes adolescentes, y llama la atención que se distribuyen de manera semejante en todas las UPA, de manera independiente a su nivel socioeconómico.

La edad gestacional y el nivel educativo muestran una gran variabilidad, según el lugar de captación de las participantes. Estos centros de atención se caracterizan por atender a la población del sistema público de salud en un área geográfica que concentra una sociedad de un nivel socioeconómico muy bajo o bajo, referido en adelante como estratos socioeconómicos 1,2 y 3 . En la tabla 1 se muestra la manera en que se ha distribuido la población en cada unidad primaria de atención.

TABLA 1

Características demográficas de las participantes

\begin{tabular}{|c|c|c|c|c|c|c|}
\hline \multirow{3}{*}{ Edad } & \multicolumn{4}{|c|}{ Unidad primaria de atención } & \multirow{2}{*}{ Total } & \multirow{3}{*}{$\begin{array}{c}\text { Kruskal- } \\
\text { Wallis } \\
p<0,05^{* *}\end{array}$} \\
\hline & 1 & 2 & \multirow{2}{*}{$\begin{array}{c}3 \\
\text { Fre }(\%)\end{array}$} & \multirow{2}{*}{$\begin{array}{c}4 \\
\text { Fre }(\%)\end{array}$} & & \\
\hline & $\frac{1}{\text { Fre }(\%)}$ & Fre $(\%)$ & & & Fre $(\%)$ & \\
\hline $14-19$ & (8) 13,11 & (8) 13,11 & (27) 44,26 & (18) 29,5 & (61) 40,67 & \multirow{5}{*}{0,794} \\
\hline $20-30$ & (5) 7,14 & (15) 21,5 & (17) 24,2 & (33) 47,2 & (70) 46,67 & \\
\hline $31-40$ & (3) 16,7 & (3) 16,7 & (10) 55,5 & (2) 11,1 & (18) 12 & \\
\hline$>40$ & $(0) 0$ & $(0) 0$ & $(0) 0$ & (1) 100 & (1) 0,66 & \\
\hline Total & 16 & 26 & 54 & 54 & 150 & \\
\hline \multicolumn{7}{|c|}{ Nivel socioeconómico } \\
\hline 1 & (3) 37,5 & (1) 12,5 & $(0) 0$ & (4) 50 & (8) 5,33 & \multirow{4}{*}{$0,000 * *$} \\
\hline 2 & (11) 13,5 & (10) 12,2 & (18) 22 & (43) 52,5 & (82) 54,67 & \\
\hline 3 & (2) 3,4 & (15) 25 & $(36) 60$ & (7) 11,6 & (60) 40 & \\
\hline Total & 16 & 26 & 54 & 54 & 150 & \\
\hline
\end{tabular}

Fuente: elaboración propia.

Se han considerado dos características de gran sensibilidad: la edad y el nivel socioeconómico, pues la literatura las ha referido como influyentes en la valoración del cuidado $(17,18)$, y puede observarse que la distribución socioeconómica es distinta $(\mathrm{p}=0,000)$ entre los lugares de atención. Mientras las UPA 1 y 4 concentran las madres de estratos 1 y 2 ; las UPA 2 y 3 , a las madres del nivel 3. Estos hallazgos pueden obedecer a la ubicación geográfica de los centros de atención.

Frente a los análisis realizados sobre la valoración que las madres tienen del cuidado profesional, la figura 1 presenta la clasificación del cuidado que la escala permite, y se observa que no hay diferencias estadísticamente significativas de la percepción que tienen las mujeres. Sin embargo, se refleja que existe una menor satisfacción de las usuarias que reciben atención en la UPA 2. 


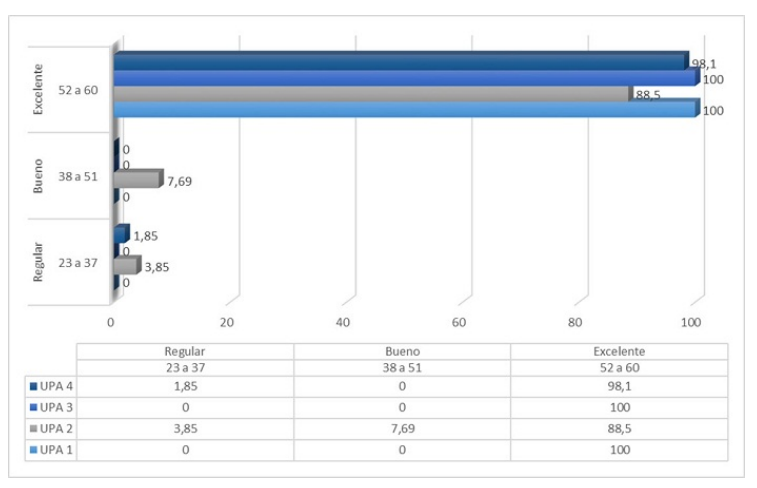

FIGURA 1

Percepción de las gestantes sobre el cuidado profesional de enfermería Fuente: elaboración propia

Cuando se observa el instrumento de manera integral, se encuentra una percepción general de las usuarias, donde el 1,33\% considera el cuidado profesional como regular; $1,33 \%$ lo considera bueno, y el 97,3\% lo evalúa como excelente. De acuerdo con la interpretación de la escala empleada, el cuidado profesional será considerado regular: cuando es "neutral”; es básicamente apático o individual, no es de persona a persona. Es bueno cuando se caracteriza como benevolente, amable y preocupado. El resultado de este encuentro es que los niveles de energía son sostenidos o aumentados. Es excelente cuando implica estar completamente presente, con la curación de amor que fluye. La personalidad se reconoce mutuamente, la atención se negocia y la intimidad profesional se produce. Esta relación favorece la libertad espiritual (14).

Estos resultados destacan un alto nivel de satisfacción con el cuidado recibido. Sin embargo, al realizar un análisis mucho más discriminado se encuentran aspectos de gran valor que deben ser contemplados. La figura 2 permite ver que la satisfacción con la habilidad de los profesionales de enfermería para ofrecer un cuidado sensible, que identifica las necesidades individuales, puede mejorarse en todos los lugares de atención, e incluso cerca de un 8\% de las mujeres que recibieron atención en la UPA 2 lo califican como regular.

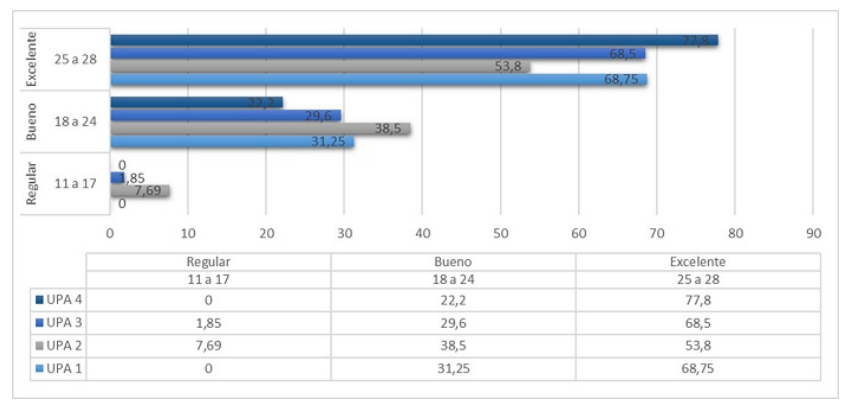

FIGURA 2

Valoración de la subescala: sanador compasivo

Fuente: elaboración propia.

Al observar el comportamiento ítem por ítem de la escala, se encuentran diferencias estadísticamente significativas evaluadas con la prueba Kruskal-Wallis en el ítem 4, que evalúa la capacidad del profesional de permitir la expresión de sentimientos de la mujer gestante $\left(\mathrm{p}=0,025^{* *}\right)$. Esta es una de las habilidades importantes del cuidado prenatal, pues es ampliamente conocido que las mujeres durante la gestación experimentan cambios emocionales importantes y alto nivel de sensibilidad. De acuerdo con la percepción de las mujeres gestantes, la UPA 3 fue el lugar de atención con la menor calificación, y la UPA, 4 la mejor calificada. En el ítem 5, que evalúa la percepción de la mujer frente a las muestras de interés por su situación personal, y en el ítem 2, que evalúa la actitud del profesional con la mujer y su gestación, se evidenciaron bajas calificaciones, pues las mujeres no lo sintieron con frecuencia en los momentos de cuidado, siendo la UPA 2 la peor calificada, y la UPA 4, la mejor calificada. Otro ítem de gran valor para la evaluación del cuidado profesional de las enfermeras es el 12, pues evalúa la percepción de las mujeres de haberse sentido cuidadas. Se sabe que cuidar es el deber ser de enfermería (19); sin embargo, la percepción de las mujeres muestra baja satisfacción en este ítem, encontrándose entre el 43,7\% y el 74\%. La subescala sanador compasivo muestra 
unos retos importantes de mejora, ya que hay una calificación general del cuidado que es considerado excelente solo por el 69,3\%. Debe resaltarse que en ninguna de las subescalas se alcanzó una puntuación deficiente.

La figura 3 muestra la evaluación que las gestantes hacen de las competencias y habilidades cognitivas de los profesionales que ofrecen el cuidado profesional. Y puede concluirse que la UPA 2 es el centro de atención donde se observa una satisfacción menor; mientras que en la UPA 4 todas las gestantes lo evalúan como excelente. Al hacer una discriminación más detallada no se encuentran diferencias estadísticamente significativas que deban ser expuestas.

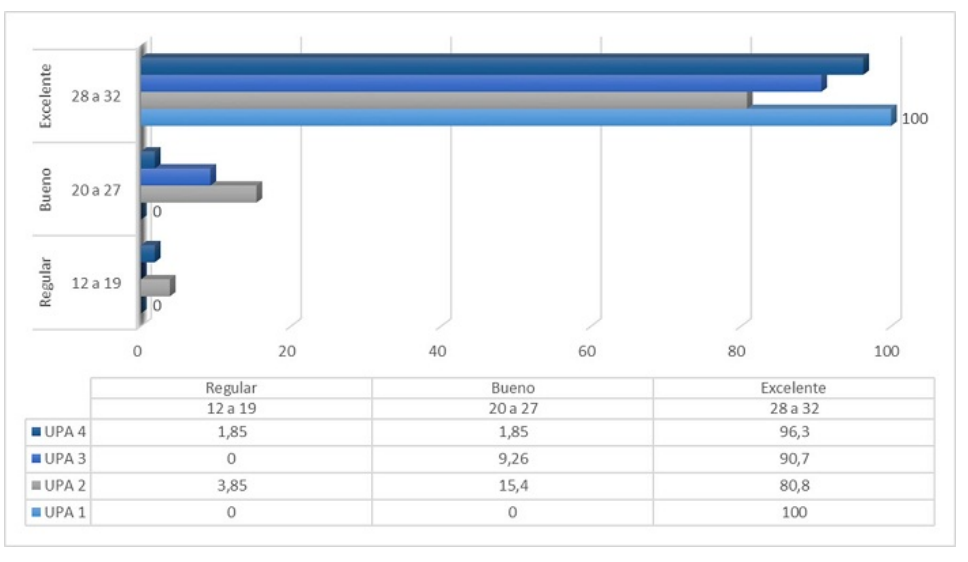

FIGURA 3

Valoración de la subescala: sanador competente

Fuente: elaboración propia

Las pruebas de correlación emplearon la prueba correlación de Spearman o por rangos, y se encontraron interesantes asociaciones. Sin embargo, la fuerza de ellas es débil en algunos casos; pero significativas o altamente significativas. Por lo anterior, los investigadores consideran importante que estas asociaciones se tengan en cuenta en futuras investigaciones y se muestran en la tabla 2.

TABLA 2

Asociación de variables del estudio

\begin{tabular}{|l|r|r|r|r|r|r|r|}
\hline \multicolumn{1}{|c|}{ Variables } & $\begin{array}{c}\text { Percepción } \\
\text { ECP }\end{array}$ & $\begin{array}{c}\text { Sanador } \\
\text { compasivo }\end{array}$ & UPA & Ítem 2 & Ítem 4 & Ítem 5 & Ítem 12 \\
\hline Edad & $0,240^{*}$ & $0,260^{* *}$ & $(-)$ & $0,343^{* *}$ & $0,207 *$ & $0,219 *$ & $0,170^{*}$ \\
\hline $\begin{array}{l}\text { Sanador } \\
\text { Compasivo }\end{array}$ & $0,956^{* *}$ & 1 & $0,193 *$ & $0,825 * *$ & $0,802^{* *}$ & $0,858^{* *}$ & $0,590^{* *}$ \\
\hline $\begin{array}{l}\text { Sanador } \\
\text { Competente }\end{array}$ & $0,846^{* *}$ & $0,697 * *$ & $(-)$ & $0,593 * *$ & $0,611 * *$ & $0,822^{* * *}$ & $0,697 * *$ \\
\hline Item 13 & $0,539 * *$ & $0,509^{* *}$ & $(-)$ & $0,488^{* * *}$ & $0,357 * *$ & $0,393 * *$ & $0,427 * *$ \\
\hline
\end{tabular}

ECP: Escala de Cuidado Profesional. ${ }^{*} \mathrm{p}<0,05 ;{ }^{* *} \mathrm{p}<0,01 ;(-)$ : no es significativa Fuente: elaboración propia.

Debe resaltarse el papel que desempeña la subescala de sanador compasivo frente a la percepción del concepto de cuidado profesional, pues se demuestra una gran fuerza en la asociación (20), siendo superior a la fuerza de la subescala de sanador competente, a pesar de tener un número inferior de ítems. Además es de gran interés ver cómo la valoración de ítems específicos, como el ítem 2, que evalúa la actitud del profesional; el ítem 4, que evalúa la habilidad de permitir la expresión de sentimientos, y el ítem 5, que valora las muestras de interés del profesional en la mujer gestantes, se relacionan fuertemente con la valoración de la subescala sanador competente, es decir, podrían afectar la percepción que tienen las mujeres gestantes de las habilidades técnicas y competencias cognitivas de los profesionales. Así mismo, es muy interesante observar que el ítem 13, que evalúa la compresión de las indicaciones, muestra una asociación moderada con la percepción de la subescala sanador compasivo. Para finalizar, futuras investigaciones deben precisar la relación que existe entre 
la edad de la mujer gestante y la percepción que tiene del cuidado compasivo, pues los resultados presentan una relación directa, donde a mayor edad, mejor percepción y viceversa. No obstante, existen argumentos teóricos que soportan estos hallazgos (17).

\section{Discusión}

Las cifras de embarazo en adolescentes superan el $40 \%$, mayor a los datos que oficialmente se reportaban para la ciudad de Bogotá en 2013, que alcanzaban el 17\% (21). Con ello es posible pensar que este fenómeno tiene alta incidencia en la localidad de Engativá, lugar de realización del estudio. Los resultados de escolaridad y estratificación socioeconómica contextualizan a las participantes en un mayor nivel de vulnerabilidad. Esta es una condición que se caracteriza por una necesidad aumentada de un trato sensible y humanizado (17). Por lo anterior, es importante que la evaluación de calidad de la atención prenatal permita evaluar la satisfacción de las gestantes con su cuidado.

En una revisión sistemática que incluye 11 investigaciones con rigurosos criterios de calidad metodológica, que evalúan la calidad del cuidado obstétrico, se evaluó la satisfacción de las gestantes en cinco de ellos. Sin embargo, no emplean instrumentos que permitan una amplia y detallada medición. Lo evalúan mediante la percepción de satisfacción o la pregunta de si volvería a elegir ese tipo de cuidado. Presentan un porcentaje de satisfacción que oscila entre el 92\% y el 94\% (3). Ello es semejante a los resultados de esta investigación, donde se encuentra que la satisfacción es superior al $97 \%$, pues las madres perciben como excelente el cuidado recibido.

Sin embargo, los resultados encontrados en los ítems 2, 4, 5 y 12 son fiel reflejo de la necesidad de trabajar en un cuidado más integral y humanizado. Investigadores como Duarte et al. (8) y Huaman et al. (10), en Suramérica, y por Mckinnon et al. (9), en Australia, presentan hallazgos semejantes. Estos autores han planteado que la capacidad del prestador del cuidado de establecer una comunicación efectiva y asertiva con la mujer gestante puede afectar positiva o negativamente la adherencia a la atención prenatal. De la misma manera, la actitud positiva desprovista de la actitud crítica, ante una evolución insatisfactoria, ante un hijo adicional o la edad de la mujer, es fundamental en la atención de la mujer gestante. Y puede ser una barrera fuerte que no permite la identificación clara de las necesidades individuales (9).

$\mathrm{Al}$ contrastar estos hallazgos con los resultados de esta investigación, podemos ver que el ítem 2, que interroga sobre la actitud del profesional con la mujer y su embarazo, presenta un bajo nivel de satisfacción que varía por lugar entre el 57\% y el 77\%; sin embargo, esta diferencia no es estadísticamente significativa. No obstante, se asocia de manera significativa con las puntuaciones de la subescala sanador competente, con la edad y con la claridad de las indicaciones que recibe la madre para la continuidad de su cuidado.

En otro estudio que empleó una metodología mixta, realizado en Londres, las mujeres igualmente apreciaron que el prestador de la atención les diera un espacio para expresar sus sentimientos, que tuvieran un estilo de conversación empático y se refirieron a las preguntas abiertas como las mejores para favorecer la identificación de sus necesidades (22). En ese mismo estudio, las mujeres manifestaron que las dificultades en la comunicación en algunas oportunidades les impidió entender las indicaciones con claridad y comprender su evolución en la gestación (22).

En esa dirección, los resultados encontrados en este estudio apoyan lo manifestado en Londres, empleando la metodología de grupos focales, pues el ítem 13 - que evalúa la claridad de la explicación de las indicaciones - se relaciona con una fuerza moderada con la percepción general de la subescala de sanador compasivo (Rho: 0,509; $\mathrm{p}<0,01)$. De la misma manera, los resultados de esta investigación presentan una correlación más fuerte entre la subescala sanador compasivo con el resultado final de la percepción del cuidado profesional, comparado con la fuerza de la relación de esta con la subescala sanador competente (Rho: 0,956 y p < 0,01 vs. Rho: 0,846 y $\mathrm{p}<0,01)$. 
Esta postura adquiere mayor solidez cuando se observa una fuerte asociación entre ítems específicos como el 2, el 4 y el 5 con la percepción general de la escala sanador competente. Así se comprende que la relación preocupada, que permite la expresión de sentimientos y mantiene una actitud positiva entre el profesional y la mujer gestante, puede influir en la percepción que ella tiene de las habilidades técnicas y cognitivas del prestador de la atención.

Resultados similares se encontraron en un estudio correlacional realizado en Reino Unido, donde la percepción de una mala relación de la mujer gestante con su prestador de atención prenatal influyó significativamente en la manera en como ellas percibían la responsabilidad, la culpa y los riesgos asumidos por el profesional a la hora de atenderlas; identificaron que son peor valorados cuando hay mala relación que cuando existe una relación positiva. Estos resultados se obtuvieron de manera independiente al riesgo, la culpa o la irresponsabilidad real y objetiva (23). Otras variables como la raza (24) y el embarazo no deseado (25) se han asociado a la percepción de la calidad del cuidado prenatal en estos dos aspectos específicos de la habilidad técnica y cognitiva y el cuidado sensible y humanizado; sin embargo, el presente estudio no dispone de datos frente a estas variables.

\section{Conclusiones}

Los resultados encontrados en la presente investigación exponen que las madres gestantes participantes perciben como excelente el cuidado que reciben de los profesionales de enfermería durante la atención prenatal. Sin embargo, análisis específicos de cada una de las dimensiones del cuidado seleccionadas muestran carencias de los profesionales para ofrecer un cuidado compasivo. De la misma manera, es evidente que el cuidado profesional tiene un componente de habilidades personales que pueden influir positiva o negativamente en la percepción de la atención. Por ello deben ser requisitos en la atención de la misma manera que los son sus habilidades cognitivas. Los profesionales de enfermería deben reforzar su trabajo personal, mejorando sus habilidades de interacción y comunicación empática, pues no son suficientes los conocimientos y las destrezas técnicas para dar un cuidado de calidad. Además, esta investigación reconoce la necesidad de que las instituciones de salud desarrollen medidas que permitan a los usuarios una valoración frecuente del servicio que reciben.

Son interesantes los resultados que contrastan las dos subescalas: sanador compasivo y competente, pues resaltan aspectos coincidentes con los hallazgos de otros autores en poblaciones, culturas y metodologías de estudio diferentes. Por ello, investigaciones futuras con muestras de mayor poder deben clarificar la existencia de aquellas relaciones que la literatura sigue planteando y confirmar o refutar las planteadas en esta investigación.

Es preciso que los profesionales que ofrecen atención prenatal sean cada día más conscientes de la exigencia de su labor y de la importancia que cobran detalles que pueden parecer poco significativos, como la escucha activa o la expresión de sentimientos; pero que son determinantes en la adherencia al control prenatal y la satisfactoria evolución de la salud materna y perinatal. Cada uno, desde su sitio de trabajo, de manera independiente a la disposición o no de los mejores recursos físicos y tecnológicos, puede hacer la diferencia.

\section{Referencias}

1. Liu CM, Chang SD, Cheng PJ. Relationship between prenatal care and maternal complications in women with preeclampsia: Implications for continuity and discontinuity of prenatal care. Taiwan J Obstet Gynecol. 2012;51(4):576-82. https://www.dx.doi.org/10.1016/j.tjog.2012.09.013.

2. Spears W, Hook L, Castillo J, Torres C. Initiation of and barriers to prenatal care use among low-income women in San Antonio, Texas. Matern Child Health J. 2010;14:133-40. https://www.doi.org/10.1007/s10995-008-0 419-0. 
3. Sheeder J, Weber K, Kabir-greher K. A review of prenatal group care literature: the need for a structured theoretical framework and systematic evaluation. Matern Child Health J. 2012;16:177-87. https://www.doi.org/10.1007 /s10995-010-0709-1

4. Jiménez Puñales S, Pentón Cortés RJ. Mortalidad perinatal: factores de riesgo asociados. Clin Invest Ginecol Obstet. 2013;42(1):2-6. https://www.doi.org/10.1016/j.gine.2013.05.003

5. Dowswell T, Carroli G, Duley L, Gates S, Gülmezoglu a M, Khan-Neelofur D, et al. Alternative versus standard packages of antenatal care for low-risk pregnancy. Cochrane Database Syst Rev. 2010;(10):CD000934. https:/ /www.doi.org/10.1002/14651858.

6. Abu-Ghanem S, Sheiner E, Sherf M, Wiznitzer A, Sergienko R, Shoham-Vardi I. Lack of prenatal care in a traditional community: Trends and perinatal outcomes. Arch Gynecol Obstet. 2012;285(5):1237-42. https://www.doi.or g/10.1007/s00404-011-2153-x.

7. UNICEF. Estrategia de UNICEF en América Latina y el Caribe para contribuir a la reducción de la morbimortalidad materna, neonatal e infantil. 2011-2015 [internet]. 2013. Disponible en: https://www.unicef.org/lac/Estrateg ia_Mortalidad_Materna_Neonatal.pdf.

8. Duarte S, Oliveira de Andrade S, Mamede MV. Maternal decision on obtaining prenatal care: A study in Brazil. Midwifery. 2011;27:160-4. https://www.doi.org/10.1016/j.midw.2009.04.008

9. Mckinnon LC, Prosser SJ, Miller YD. What women want: qualitative analysis of consumer evaluations of maternity care in Queensland, Australia. BMC Pregnancy Childbirth [internet]. 2014;14(366). Disponible en: https://w ww.biomedcentral.com/1471-2393/14/366.

10. Huaman LS, Blumenthal P, Sarnquist CC. Factors influencing women's decision to seek antenatal care in the Andes of Peru. Matern Child Health J. 2013;17:1112-8. https://www.doi.org/10.1007/s10995-012-1113-9.

11. Morestin F, Bicaba A, Sermé JDD, Fournier P. Evaluating quality of obstetric care in low-resource settings: Building on the literature to design tailor-made evaluation instruments - an illustration in Burkina Faso. BMC Health Serv Res. 2010;10(20). https://www.doi.org/10.1186/1472-6963-10-20.

12. Hernández Betancur AM, Vásquez-Truisi ML. El cuidado de enfermería comprometido: motor en la satisfacción de la gestante durante el control prenatal. Univ Salud. 2015;17(1):80-96.

13. Hernández Sampieri R, Fernández Collado C, Baptista Lucio MDP. Metodología de la investigación [internet]. 5a ed. México: McGraw Hill; 2014. Disponible en: https://www.casadellibro.com/libro-metodologia-de-la-invest igacion-5-ed-incluye-cd-rom/9786071502919/1960006.

14. Vesga Gualdrón LM, Ruiz CH. Validez y confiabilidad de una escala de cuidado profesional en español. Av Enfemr. 2016;34(1):69-78. https://www.dx.doi.org/10.15446/av.enferm.v34n1.44488

15. Bassan H, Uliel-sibony S, Katsav S, Farber M, Tauman R. Maternal sleep disordered breathing and neonatal outcome. 2016;18(January):45-8.

16. de Figueiredo FP, Parada AP, Cardoso VC, Batista RFL, da Silva AAM, Barbieri MA, et al. Postpartum depression screening by telephone: a good alternative for public health and research. Arch Womens Ment Health. 2015;18(3):547-53. https://www.doi.org/10.1007/s00737-014-0480-1

17. Muñoz LA, Vollrath A, Bonatti C. Vivenciando la maternidad en contextos de vulnerabilidad social: un enfoque comprensivo de la fenomenología social. Rev Latino-Am Enferm [internet]. 2013;21(4). Disponible en: https ://www.eerp.usp.br/rlae.

18. Groth SW, Holland ML. Eighteen years after pregnancy adolescent gestational weight gain still affects body mass index. J Obstet Gynecol Neonatal Nurs [internet]. 2013;42(s1):S79-80. Disponible en: https://www.doi.wiley .com/10.1111/1552-6909.12168.

19. Fawcett J. Analisys and evaluation of contemporary nursing knowledge. 3a ed. Philadelphia: F. A. Davis Company; 2013.

20. Martínez Ortega RM. El coeficiente de correlación de los rangos de Spearman caracterización. Rev Haban Cienc Méd. 2009;VIII(2):1-19. 
21. Departamento Administrativo Nacional de Estadística [internet]. [Citado 2015 jul 28]. Disponible en: https://www.dane.gov.co/index.php/esp/poblacion-y-demografia/nacimientos-y-defunciones/118-demog raficas/estadisticas-vitales/4647-nacimientos-2013.

22. Raine R, Cartwright M, Richens Y, Mahamed Z, Smith D. A qualitative study of women's experiences of communication in antenatal care: Identifying areas for action. Matern Child Health J. 2010;14(4):590-9. https ://www.doi.org/10.1007/s10995-009-0489-7.

23. Lawton R, Gardner P, Plachcinski R. Using vignettes to explore judgements of patients about safety and quality of care: The role of outcome and relationship with the care provider. Heal Expect. 2011;14(3):296-306.

24. Ruwe M, Capitman J, Bengiamin M, Soto T. A systematic review and meta-analysis of racial disparities in prenatal care in California: How much? Does insurance matter? Soc Work Public Health. 2010;25(6):550-71.

25. Erol N, Durusoy R, Ergin I, Döner B, Ciçeklioğlu M. Unintended pregnancy and prenatal care: a study from a maternity hospital in Turkey. Eur J Contracept Reprod Health Care. 2010;15(4):290-300.

\section{Notas}

*Artículo original de investigación

Conflictos de interés: Los autores declaran no tener conflictos de interés.

Financiamiento: Ninguno

\section{Licencia Creative Commons CC BY 4.0}

Cómo citar: Vesga Gualdrón LM, Ruiz de Cárdenas CH. Percepción que tienen las gestantes sobre el cuidado de enfermería en la atención prenatal. Investig Enferm Imagen Desarr. 2019;21(1). https://www.doi.org/10.11144/Javeriana.ie21-1.ptgc 